\title{
Comparison of opioid free anaesthesia with opioid anaesthesia on postoperative shivering in morbidly obese patients scheduled for bariatric surgery
}

\section{J. Persyn, P Van Lancker, J Mulier. Department of Anaesthesiology, AZ Sint Jan Brugge-Oostende}

\section{Background}

Post-anaesthetic shivering (PAS) occurs frequently. The primary cause is believed to be peri-operative hypothermia caused by anaesthetic induced vasodilatation and inhibition of thermoregulation while exposed to a cold environment.

Research has shown that several drugs including the opioids meperidine and tramadol, clondine, dexmedetomidine, ondansetron and ketamine treat and prevent shivering by reducing the shivering threshold temperature.

In addition, several studies concluded that patients had significant less shivering and less discomfort of feeling cold when given opioid free anaesthesia.

\section{Research questions}

- Does opioid free anaesthesia (OFA) has a significant impact on post operative shivering as compared to opioid anaesthesia (OA)?

- Does OFA has a significant effect on decrease in core temperature as compared to OA?

\section{Methods}

-50 patients undergoing bariatric surgery, randomly assigned to either the OFA group $(n=25)$ or the OA group $(n=25)$

-Core body temperature registered with the Spot On system (interval of 15 minutes)

- A fixed induction - maintenance protocol for both groups -OFA group

-OA group:

-Period and intensity of shivering documented by specified members in the recovery room (PACU).
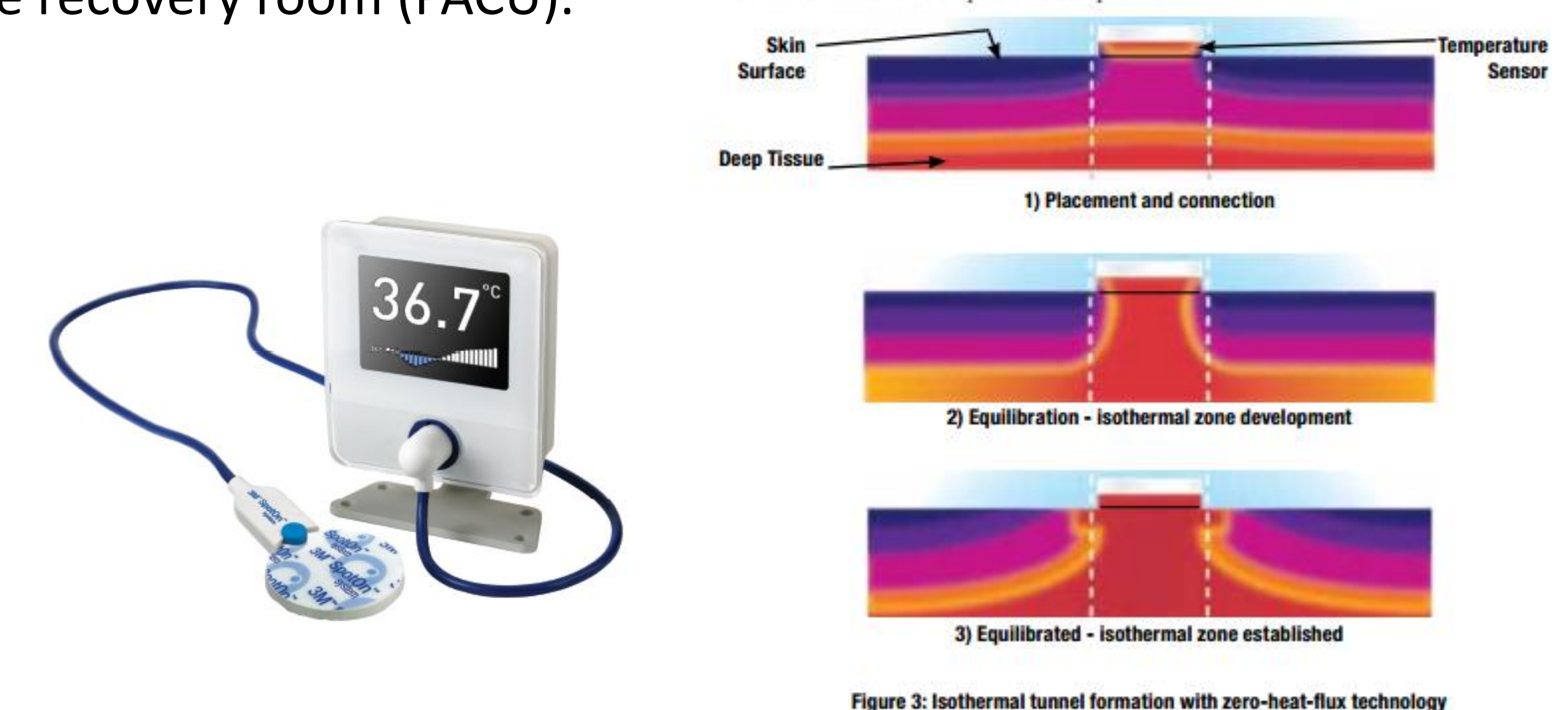

\section{Results}

Table 1: Descriptive: Patient data

\begin{tabular}{|lll|}
\hline & OFA $(\mathbf{n = 2 5})$ & OA $(\mathbf{n = 2 5})$ \\
\hline $\begin{array}{l}\text { Sex } \\
\text { (female/total) }\end{array}$ & $20 / 25$ & $17 / 25$ \\
Age & 41 jaar & 42 jaar \\
BMI & 39.39 & 38.24 \\
Diabetus & $1 / 25$ & $2 / 25$ \\
OSAS & $6 / 25$ & $4 / 25$ \\
$\begin{array}{l}\text { Body temperature } \\
\text { pre-induction }\end{array}$ & $36.7^{\circ} \mathrm{C}$ & $36.7^{\circ} \mathrm{C}$ \\
\hline
\end{tabular}

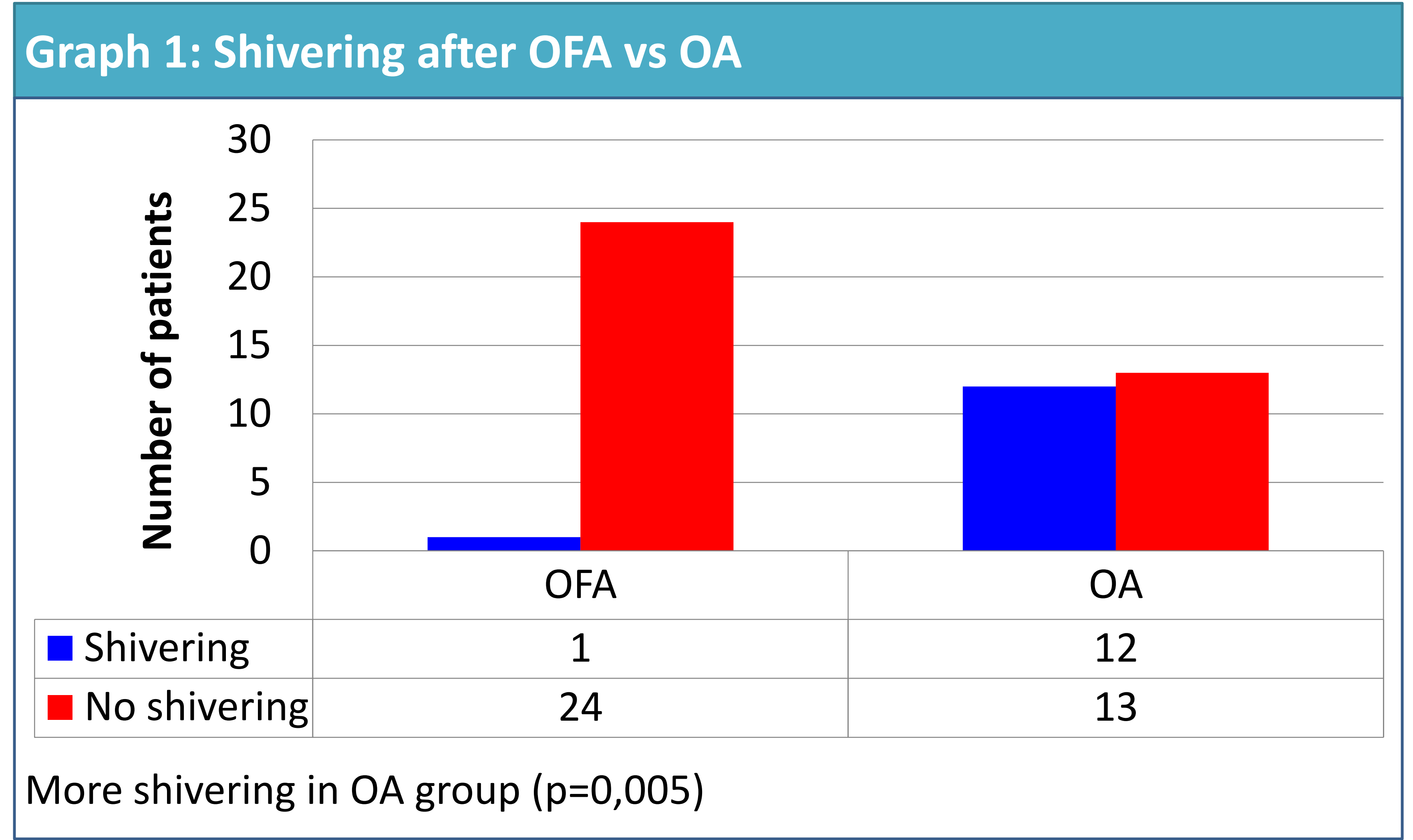

\begin{tabular}{|c|c|c|c|}
\hline & OFA $(n=25)$ & $O A(n=25)$ & T-test: $p$ \\
\hline $\begin{array}{l}\text { Temperature } \\
\text { pre-induction }\end{array}$ & $\begin{array}{l}36.7^{\circ} \mathrm{C} \\
\text { (SD 0.12) }\end{array}$ & $\begin{array}{l}36.7^{\circ} \mathrm{C} \\
\text { (SD 0.09) }\end{array}$ & \\
\hline $\begin{array}{l}\text { Mean } \\
\text { temperature } \Delta / \mathrm{h} \\
\text { Preinduction-extubation }\end{array}$ & $\begin{array}{c}0.47^{\circ} \mathrm{C} / \text { hour } \\
\quad(\text { SD 0.07) }\end{array}$ & $\begin{array}{l}0.54^{\circ} \mathrm{C} / \text { hour } \\
\quad(\mathrm{SD} 0.07)\end{array}$ & 0.433 \\
\hline $\begin{array}{c}\text { Mean } \\
\text { temperature } \Delta / \mathrm{h} \\
\text { Induction-extubation }\end{array}$ & $\begin{array}{c}0.39^{\circ} \mathrm{C} / \text { hour } \\
(\text { SD 0.05) }\end{array}$ & $\begin{array}{c}0.46^{\circ} \mathrm{C} / \text { hour } \\
\quad(\mathrm{SD} 0.07)\end{array}$ & 0.392 \\
\hline
\end{tabular}

\begin{tabular}{|c|c|c|c|c|c|c|}
\hline & B & S.E. & Wald & $\mathbf{p}$ & $\operatorname{Exp}(B)$ & Conf. Int \\
\hline OFA vs OA & 3.238 & 1.141 & 8.058 & 0.005 & 25.480 & {$[2.725 ; 238.288]$} \\
\hline $\begin{array}{l}\text { Length } \\
\text { procedure }\end{array}$ & -0.11 & 0.17 & 0.382 & 0.537 & 0.989 & {$[0.957 ; 1.023]$} \\
\hline $\begin{array}{l}\text { Mean temp } \\
\text { decrease }\end{array}$ & .732 & 1.389 & 0.278 & 0.598 & 2.08 & {$[0.137 ; 31.629]$} \\
\hline $\begin{array}{l}\text { Preinduction } \\
\text { temp }\end{array}$ & 0.285 & 0.830 & 0.118 & 0.731 & 1.33 & {$[0.262 ; 6.759]$} \\
\hline Constant & -16.158 & 30.472 & 0.281 & 0.596 & 0.000 & \\
\hline
\end{tabular}

\section{Conclusion}

A significant difference in post-anaesthetic shivering was found between OFA and OA groups, which could not be related to the intra- and perioperative decrease in core body temperature.

\section{Table 2: Descriptive: External factors}

\begin{tabular}{|lll|}
\hline & OFA $(\mathbf{n}=\mathbf{2 5})$ & OA $(\mathbf{n}=\mathbf{2 5})$ \\
\hline Duration procedure & $110.8 \mathrm{~min}$ & $118.2 \mathrm{~min}$ \\
& $(\mathrm{SD} 4.66)$ & $(\mathrm{SD} 5.47)$ \\
Temp operating room & $17.24^{\circ} \mathrm{C}$ & $17.28^{\circ} \mathrm{C}$ \\
& $(\mathrm{SD} \mathrm{0.12)}$ & $(\mathrm{SD} 0.15)$ \\
\hline
\end{tabular}

